\title{
THE EFFECTS OF AUTUMN AND SPRING SOWING ON YIELD, OIL AND MORPHINE CONTENTS IN THE TURKISH POPPY (Papaver somniferum L.) CULTIVARS
}

\author{
Nimet KARA \\ Suleyman Demirel University, Faculty of Agriculture, Department of Field Crops, Isparta, TURKEY \\ Corresponding author: nimetkara@sdu.edu.tr
}

Received: 12.08.2016

\begin{abstract}
The poppy is a plant that can be planted in the autumn and spring seasons. The purpose of the research was to investigate the effects of autumn and spring sowing on capsule yield, seed yield, yield components, oil content and morphine content of fifteen poppy cultivars that have been recently registered under Isparta conditions. The experiment was arranged according to the randomized complete-block design with three replicates during the years of 2012/13 and 2013/14. The results of the research showed that the differences among capsule yields, seed yields, yield components and morphine contents of the cultivars were statistically significant in both sowing times and years. The highest and the lowest values of plant height, capsules number, capsule width, capsule length, 1000 seed weight and oil yield of the poppy cultivars varied according to the cultivars, the sowing times and the years. The capsule yield and seed yield of the poppy cultivars in the autumn sowing varied between 416.7-1043.3 kg ha-1 and 523.5-1276.3 kg ha-1 in the first year, between 465.3-1375.6 kg ha-1 and 596.7-1520.4 $\mathrm{kg} \mathrm{ha}^{-1}$ in the second year, respectively. In the spring sowing, the capsule yield and seed yield of the poppy cultivars varied between $204.3-455.7 \mathrm{~kg} \mathrm{ha}^{-1}$ and $286.0-573.0 \mathrm{~kg} \mathrm{ha}^{-1}$ in the first year, between 497.2-830.7 $\mathrm{kg} \mathrm{ha}^{-1}$ and 614.3-962.7 $\mathrm{kg} \mathrm{ha}^{-1}$ in the second year, respectively. The highest oil content was determined in the Zaferyolu cultivar in both years and sowing times. The morphine content of the poppy cultivars varied between $0.47-1.00 \%$ in the autumn sowing and between $0.45-0.97 \%$ in the spring sowing, respectively. The higher values in the autumn sowing were obtained than the spring sowing in all of the characteristics investigated in both years, except for the oil content. Considering the present results, TMO-3, Ofis-4, Ofis-8, Tinaztepe and Zaferyolu cultivars were advised in the autumn sowing and the TMO-1, Ofis-8 and Afyon-95 cultivars in the spring sowing because of their higher capsule, seed and oil yields. In point of the morphine contents, the TMO-3, TMO-T, Ofis-3, Ofis-8 and Bolvadin-95 cultivars were advised.
\end{abstract}

Key words: Cultivar, morphine content, oil yield, poppy, seed yield

\section{INTRODUCTION}

The poppy (Papaver somniferum L.) is used in medicine, in food, in cosmetic, and the paint industries because it contains alkaloids of capsules, and for the oil and protein content of the seeds (Gumuscu and Arslan, 2008). The poppy capsules contain thirty different alkaloids, mainly morphine, codeine, thebaine, noscapine and papaverine, and are used to produce semi-synthetic active pharmaceuticals (Skalicky et al., 2014). The poppy mostly cultivated in countries such as India, Japan, China, France, Spain. In Turkey, Kütahya, Afyon, Burdur, Isparta, Balıkesir, Konya, Amasya, Çorum, Tokat, Manisa, Uşak, Denizli and Eskişehir provinces are main poppy production areas (Karabuk, 2012). Most poppy cultivation takes place in Turkey and India. The poppy is cultivated in an area of 101874 hectares in world (FAO, 2015), and it is cultivated on an area of 61591 hectares, with an annual production of 30730 tons and an average yield of $500 \mathrm{~kg} \mathrm{ha}^{-1}$ in Turkey (TUIK, 2015). More than $50 \%$ of the total sowing areas in the world is performed in Turkey according to the data from 2015 year (FAO, 2015). However, the seed yield in per hectare and morphine content of the capsule is low in Turkey when compared to other poppy producer countries. Recently, the ten poppy cultivars with high morphine content were registered by the Soil Products Office. The poppy quality is mainly evaluated with its oil and morphine content. Poppy varieties with high alkaloid contents are used for medicinal purpose, and varieties with low alkaloid contents and high seed yielding are used for food production (Prajapati et al., 2002). Arslan et al. (2000) reported that the morphine and oil contents of the poppy populations in Turkey varied with $0.25-0.89 \%$ and $38.65-$ $53.38 \%$, respectively. Erdemoglu et al. (2002) stated that the morphine contents of the poppy capsules obtained from the different provinces in Turkey varied with 0.093 $0.263 \%$. Karadavut and Arslan (2006) reported that the 
plant height, capsule number, capsule yield, seed yield and morphine content of the poppy cultivars varied between 22.21-99.71 cm, 1.01-6.17 capsules, 0.30-6.48 $\mathrm{g} \mathrm{plant}^{-1}$, $0.26-11.66 \mathrm{~g} \mathrm{plant}{ }^{-1}$ and $0.22-1.225 \%$, respectively. Gumuscu et al. (2008) determined that the morphine contents of the different poppy lines which were autumn cultivated varied between $0.110-1.140 \%$. Koc et al. (2014) reported that the capsule yield, seed yield and morphine content of the autumn poppy cultivars varied with 420 $980 \mathrm{~kg} \mathrm{ha}{ }^{-1}, 720.0-1170.0 \mathrm{~kg} \mathrm{ha}{ }^{-1}$ and $0.45-1.30 \%$, respectively. Boydak and Kavurmaci (2015) stated that the capsule number, capsule yield, seed yield, capsule width, capsule size and morphine rate of poppy cultivars varied with 3.03-4.47 capsules, 92.9-140.5 kg ha-1, 577.4$1046.4 \mathrm{~kg} \mathrm{ha}^{-1}, 4.33-4.44 \mathrm{~mm}, 3.38-3.91 \mathrm{~mm}$ and $0.23-$ $0.83 \%$, respectively. The poppy is a plant that can be planted in the autumn and spring seasons. The poppy can be planted as a spring sowing when there is no moisture in the soil or due to some reasons. The yield and quality characteristics of the poppy are affected by agronomic practices, soil and climatic conditions (Jaszberenyi and Nemeth, 2012; Skalicky et al., 2014). Isparta is an area, authorized for poppy cultivation by the Soil Products Office in Turkey. Although the poppy is cultivated by a considerable number of producers in Isparta, there is no comprehensive study regarding the adaptation of the new poppy cultivars in the region. To author's knowledge, besides, there is no data on the characteristics of poppy cultivars, adopted by region producers. Therefore, the aim of the research was to determine Turkish poppy cultivars with high-capsule/seed yielding, containing high oil and morphine at different sowing times under Isparta conditions.

\section{MATERIALS AND METHOD}

The field experiment was set up in a randomized complete block design in triplicates as the autumn and spring sowing in 2012/13 and 2013/14 growing seasons in Isparta ecological conditions $\left(37^{\circ} 45^{\prime} \mathrm{N}\right.$ latitude, $30^{\circ} 33^{\prime} \mathrm{E}$ longitude and $1050 \mathrm{~m}$ altitude). The Isparta province shows typical continental climate with cold and snowy winters and dry and mild-temperate summer in the Southwestern Anatolia Region. In the research, fifteen registered poppy cultivars were obtained from the Afyonkarahisar-Bolvadin Poppy Alkaloid Office and the Eskişehir Western Transitional Zone Agricultural Research Institute (Table 1). Generally, the cultivars used in the research are new varieties that have been recently registered in Turkey.

Table 1. The cultivars used in experiments and their obtained office / Institute

\begin{tabular}{ll}
\hline Cultivars & Office / Institute \\
\hline TMO-1 & \\
TMO-2 & \\
TMO-3 & \\
TMO-T & \\
Ofis-3 & \\
Ofis-4 & Afyonkarahisar-Bolvadin Poppy and Alkaloid Office \\
Ofis-8 & \\
Ofis-95 & \\
Ofis-96 & \\
Afyon-95 & \\
Bolvadin-95 & \\
\hline Tinaztepe & \\
Anayurt & Eskişehir Western Transitional Zone Agricultural Research Institute \\
Kemerkaya & \\
Zaferyolu & \\
\hline
\end{tabular}

The sowing times: i) Autumn sowing was sown on the 5 and 9 October in the first and the second growing years, respectively. ii) Spring sowing was sown on the 1 and 6 of March in the first and the second years, respectively. The spacing used was $0.45 \times 0.15 \mathrm{~m}$ and the plot length was 5 $\mathrm{m}\left(5 \mathrm{x} 2.7=13.5 \mathrm{~m}^{2}\right)$ with 6 rows. The seeds were sown at about a $0.5-1 \mathrm{~cm}$ depth using a dibbler. After the emergence of the poppy seeds, the weeds were hand-hoed as appeared and one seedling was left.

All the necessary cultural practices were applied identically to all the cultivars in both years and sowing times. The experiments were not irrigated at any growing

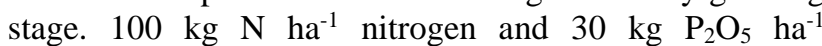
fertilizers were applied made from ammonium sulfate
(21\%) and triple super phosphate (43-46\%), respectively. Nitrogen was applied in two equal portions at sowing and at plant height $10-15 \mathrm{~cm}$ stages, respectively whereas the phosphorous fertilization was made at the sowing (Koc et al., 2012).

\section{Climatic data of the experimental area}

The climatic data for the 2012/13 and 2013/14 growing periods are shown in Table 1. During the vegetation period (from October to end of August) in $2012 / 13$ and in 2013/14, there was a total precipitation of 555.0 and $579.9 \mathrm{~mm}$, an average temperature of 12.8 and $12.1{ }^{\circ} \mathrm{C}$, and an average humidity of 58.8 and $58.3 \%$ (Table 2), respectively. 
Table 2. Some climate data of the experiment area in growing seasons*

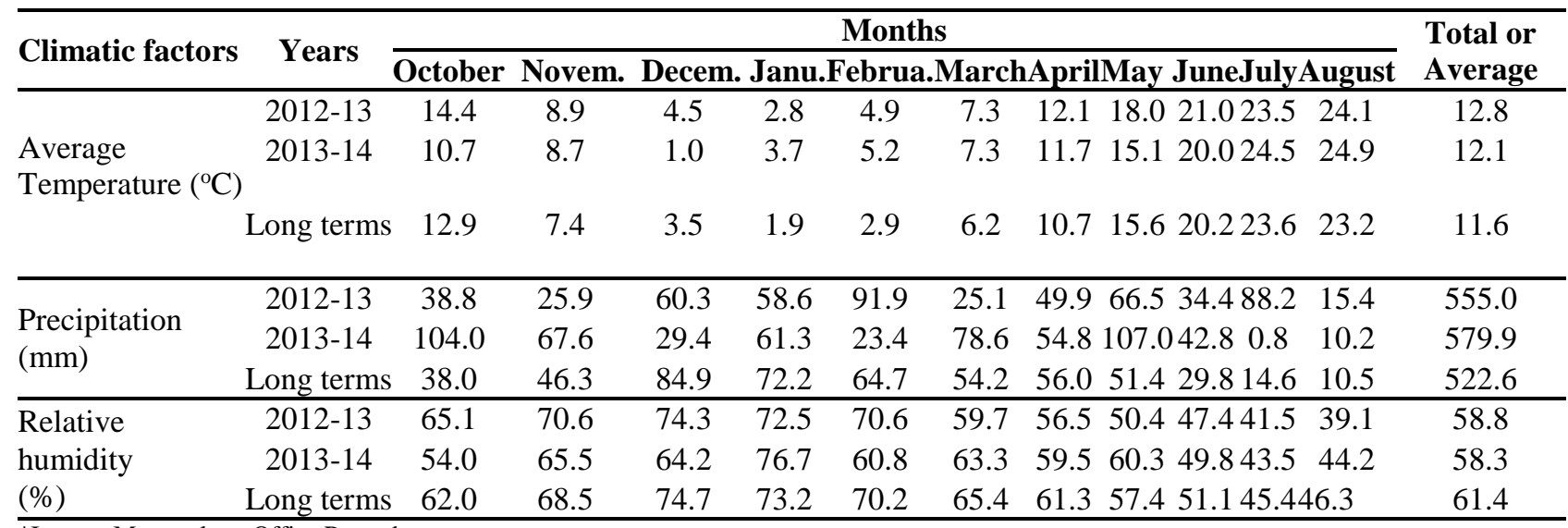

*Isparta Meteorology Office Records

\section{Soil structure}

In the years 2012/13 and 2013/14, soil at a depth of 60 $\mathrm{cm}$ was low in organic matter $(1.80 \%$ and $1.66 \%$, respectively), alkaline ( $\mathrm{pH} 7.5$ and 7.8 , respectively) limey (9.67\% and $9.89 \% \mathrm{CaCO}_{3}$, respectively), and clay-loamy.

\section{Yield and its components}

The capsules from 4 rows in the center of each plot in the full ripeness period according to the maturity stage of the cultivars were manually harvested. The capsule yield $\left(\mathrm{kg} \mathrm{ha}^{-1}\right)$, seed yield $\left(\mathrm{kg} \mathrm{ha}^{-1}\right)$, plant height $(\mathrm{cm})$, capsule number (capsule), capsule width $(\mathrm{mm})$, capsule length $(\mathrm{mm})$ and 1000 seed weight $(\mathrm{g})$ were determined as described by Karabuk (2012). The capsule yield and seed yield were calculated by multiplying by $10000 /$ plot sizes $\left(\mathrm{m}^{2}\right)$.

\section{Crude oil content analysis}

The poppy seeds were dried for 24 hours at $70{ }^{\circ} \mathrm{C}$ and $5 \mathrm{~g}$ of powdered poppy samples from each cultivar were extracted with $n$-hexan for 6 hours using the soxhlet apparatus (Buchi Universal Extraction System B-811, Germany) for determining the fixed oil content (\%).

\section{Morphine content analysis}

The capsule of the poppy was dried for 24 hours at 70 ${ }^{\circ} \mathrm{C}$, and the capsules were powered by grinding. The morphine content was analyzed according to the spectrophotometric method of the AfyonkarahisarBolvadin Poppy and Alkaloid Office.

\section{Statistical analysis}

The analysis of the variance (ANOVA) of the obtained data was analyzed by using the Jump 5.0 statistics package program. The significant differences between the means were compared according to the DUNCAN multiple range test.

\section{RESULTS}

Mean values of capsule yield, seed yield, some yield components, oil content, oil yield and morphine content of the poppy cultivars tested according to the sowing times and years were shown in Tables 3,4 and 5. In the research, the differences between the years (except for 1000-seed weight and oil content), cultivars (except for the plant height of the spring sowing in both years) and the sowing times (except for the capsule length in the first years, and the oil content in the both years) were found to be statistically significant for both years (Table 3, 4, and $5)$.

\section{Capsule yield, seed yield and their components}

The capsule and seed yields of the poppy cultivars varied with years, sowing times and varieties. The mean capsule and seed yield in the second year $\left(786.7 \mathrm{~kg} \mathrm{ha}^{-1}\right.$ and $933.8 \mathrm{~kg} \mathrm{ha}^{-1}$, respectively) was higher than the yield in the first year $\left(531.6 \mathrm{~kg} \mathrm{ha}{ }^{-1}\right.$ and $655.8 \mathrm{~kg} \mathrm{ha}^{-1}$, respectively). These differences resulted from the higher rainfall in the spring season of the plant in the second year according to the first year (Table 2).

In comparison to the sowing times, the mean capsule and seed yield in the autumn sowing was significantly higher than the spring sowing in both years. In the autumn and spring sowing, capsule yield of poppy cultivars varied between $1045.6 \mathrm{~kg} \mathrm{ha}^{-1}$ (T1naztepe)-416.7 $\mathrm{kg} \mathrm{ha}^{-1}$ (Bolvadin-95) and $455.7 \mathrm{~kg} \mathrm{ha}^{-1}$ (Ofis-8)- $204.3 \mathrm{~kg} \mathrm{ha}^{-1}$ (TMO-2) in the first year, $1375.6 \mathrm{~kg} \mathrm{ha}^{-1}$ (Ofis-8)-465.3 $\mathrm{kg} \mathrm{ha}^{-1}$ (Anayurt) and $830.7 \mathrm{~kg} \mathrm{ha}^{-1}$ (Ofis-8)- $501.6 \mathrm{~kg} \mathrm{ha}^{-1}$ (Ofis-96) in the second year (Table 4), respectively. In the autumn and spring sowing, the seed yield of the poppy cultivars varied between $1276.3 \mathrm{~kg} \mathrm{ha}^{-1}$ (Ofis-4)-523.5 kg $\mathrm{ha}^{-1}$ (Bolvadin-95) and $573.0 \mathrm{~kg} \mathrm{ha}^{-1}$ (Ofis-8)-286.0 kg ha${ }^{1}$ (TMO-2) in the first year, $1520.4 \mathrm{~kg} \mathrm{ha}^{-1}$ (Ofis-8)-596.7 $\mathrm{kg} \mathrm{ha}^{-1}$ (Anayurt) and $962.7 \mathrm{~kg} \mathrm{ha}^{-1}$ (Ofis-8)-614.3 $\mathrm{kg} \mathrm{ha}^{-1}$ (Ofis-96) in the second year (Table 4), respectively. The highest and lowest values of the yield components including the plant height, capsule number, capsule width, capsule length and 1000 seed weight of the poppy cultivars varied according to the varieties, the sowing times, and the years (Table 3 ). 
Table 3. The plant height, the number of capsule, capsule width and capsule length of poppy cultivars

\begin{tabular}{|c|c|c|c|c|c|c|c|c|}
\hline \multirow{3}{*}{$\begin{array}{l}\text { Cultivars / } \\
\text { sowing times }\end{array}$} & \multicolumn{4}{|c|}{ Plant height $(\mathrm{cm})$} & \multicolumn{4}{|c|}{ The number of capsule (capsule) } \\
\hline & \multicolumn{2}{|c|}{ 2012-13 } & \multicolumn{2}{|c|}{ 2013-14 } & \multicolumn{2}{|c|}{ 2012-13 } & \multicolumn{2}{|c|}{ 2013-14 } \\
\hline & Autumn & Spring & Autumn & Spring & Autumn & Spring & Autumn & Spring \\
\hline TMO-1 & $100.9 \mathrm{a}-\mathrm{d}$ & 66.1 & $128.2 \mathrm{ab}$ & 99.2 & 4.40efg & $1.46 \mathrm{de}$ & $4.33 \mathrm{ef}$ & $4.46 \mathrm{ab}$ \\
\hline TMO-2 & $93.2 \mathrm{de}$ & 57.9 & $114.5 \mathrm{~cd}$ & 102.3 & $4.46 \mathrm{~d}-\mathrm{g}$ & $1.96 \mathrm{cde}$ & $5.13 \mathrm{~b}-\mathrm{e}$ & $3.40 \mathrm{bcd}$ \\
\hline TMO-3 & 90.3 ef & 60.1 & $120.5 b c$ & 97.6 & $5.68 \mathrm{bcd}$ & $2.10 \mathrm{bcd}$ & $5.03 \mathrm{cde}$ & $2.96 \mathrm{~cd}$ \\
\hline TMO-T & $102.4 \mathrm{a}-\mathrm{d}$ & 65.0 & $115.5 \mathrm{~cd}$ & 101.7 & $5.26 b-f$ & $2.13 \mathrm{bcd}$ & $5.60 \mathrm{bcd}$ & $3.27 \mathrm{bcd}$ \\
\hline Ofis-3 & $82.9 \mathrm{f}$ & 58.5 & $102.9 \mathrm{e}$ & 96.9 & $4.06 \mathrm{fg}$ & $1.90 \mathrm{cde}$ & $4.50 \mathrm{def}$ & $2.43 \mathrm{~d}$ \\
\hline Ofis-4 & $96.2 \mathrm{cde}$ & 59.5 & $136.1 \mathrm{a}$ & 99.7 & $5.96 \mathrm{bc}$ & $1.36 \mathrm{e}$ & $4.96 \mathrm{cde}$ & $3.43 \mathrm{bcd}$ \\
\hline Ofis-8 & $104.4 \mathrm{abc}$ & 61.1 & $118.2 \mathrm{c}$ & 98.3 & $5.60 \mathrm{~b}-\mathrm{e}$ & $2.83 \mathrm{a}$ & $5.76 \mathrm{~b}$ & $3.46 \mathrm{bcd}$ \\
\hline Ofis-95 & $106.1 \mathrm{ab}$ & 59.6 & $116.0 \mathrm{~cd}$ & 104.2 & $5.26 b-f$ & $1.83 \mathrm{cde}$ & $4.76 c-f$ & $4.00 \mathrm{bc}$ \\
\hline Ofis-96 & $98.5 \mathrm{~b}-\mathrm{e}$ & 51.5 & $117.3 \mathrm{c}$ & 99.4 & $6.26 \mathrm{ab}$ & $1.93 \mathrm{cde}$ & $6.30 \mathrm{a}$ & $2.56 \mathrm{~d}$ \\
\hline Afyon-95 & $99.0 \mathrm{~b}-\mathrm{e}$ & 67.3 & $108.1 \mathrm{de}$ & 100.1 & $5.66 \mathrm{bcd}$ & $2.73 \mathrm{ab}$ & $6.13 \mathrm{a}$ & $3.53 \mathrm{bcd}$ \\
\hline Bolvadin-95 & $81.1 \mathrm{f}$ & 66.7 & $79.1 \mathrm{f}$ & 100.9 & $3.46 \mathrm{~g}$ & $2.86 \mathrm{a}$ & $3.70 \mathrm{f}$ & $3.26 \mathrm{bcd}$ \\
\hline Tinaztepe & $109.4 \mathrm{a}$ & 72.2 & $136.3 \mathrm{a}$ & 99.4 & $7.36 \mathrm{a}$ & $1.47 \mathrm{de}$ & $5.62 \mathrm{bcd}$ & $5.40 \mathrm{a}$ \\
\hline Anayurt & $103.3 \mathrm{abc}$ & 71.1 & $120.5 b c$ & 101.4 & $5.06 b-f$ & $1.73 \mathrm{cde}$ & $3.56 \mathrm{f}$ & $2.80 \mathrm{~cd}$ \\
\hline Kemerkaya & $101.3 \mathrm{a}-\mathrm{d}$ & 60.5 & $123.4 b c$ & 99.3 & $4.80 \mathrm{c}-\mathrm{f}$ & $1.86 \mathrm{cde}$ & $5.15 b-e$ & $2.67 d$ \\
\hline Zaferyolu & $101.2 \mathrm{a}-\mathrm{d}$ & 62.1 & $134.4 \mathrm{a}$ & 100.3 & $5.46 \mathrm{~b}-\mathrm{e}$ & $2.33 \mathrm{abc}$ & $5.40 \mathrm{~b}-\mathrm{e}$ & $2.40 \mathrm{~d}$ \\
\hline Mean $_{\text {Sowing times }}$ & $98.0 \mathrm{~A}$ & $63.3 \mathrm{~B}$ & $118.1 \mathrm{~A}$ & $99.72 \mathrm{~B}$ & $5.25 \mathrm{~A}$ & $2.04 \mathrm{~B}$ & $5.12 \mathrm{~A}$ & $3.33 \mathrm{~B}$ \\
\hline Years & \multicolumn{2}{|c|}{80.6 B } & \multicolumn{2}{|c|}{$108.9 \mathrm{~A}$} & \multicolumn{2}{|c|}{$3.6 \mathrm{~B}$} & \multicolumn{2}{|c|}{$4.2 \mathrm{~A}$} \\
\hline M.square Cultivar & 195.476 & 61.42 & 631.39 & 16.729 & 2.720 & 0.735 & 2.569 & 1.972 \\
\hline$F_{\text {value }}$ Cultivar & $10.68 * *$ & $1.49^{\mathrm{ns}}$ & 39.24 & $0.74^{\mathrm{ns}}$ & $8.71 * *$ & $8.42 * *$ & $8.70 * *$ & $6.46^{* *}$ \\
\hline C.V $(\%)$ & 4.36 & 10.15 & 3.39 & 4.76 & 10.63 & 4.44 & 10.59 & 6.56 \\
\hline
\end{tabular}

\begin{tabular}{|c|c|c|c|c|c|c|c|c|}
\hline \multirow{3}{*}{$\begin{array}{l}\text { Cultivars / } \\
\text { sowing times }\end{array}$} & \multicolumn{4}{|c|}{ Capsule width $(\mathrm{mm})$} & \multicolumn{4}{|c|}{ Capsule length $(\mathrm{mm})$} \\
\hline & \multicolumn{2}{|c|}{$2012-13$} & \multicolumn{2}{|c|}{ 2013-14 } & \multicolumn{2}{|c|}{ 2012-13 } & \multicolumn{2}{|c|}{$2013-14$} \\
\hline & Autumn & Spring & Autumn & Spring & Autumn & Spring & Autumn & Spring \\
\hline TMO-1 & $41.30 \mathrm{abc}$ & $24.39 \mathrm{~g}$ & $46.75 a b c$ & $39.06 \mathrm{a}$ & $40.66 \mathrm{cde}$ & $36.17 \mathrm{e}$ & $36.12 \mathrm{bcd}$ & $29.73 a-d$ \\
\hline TMO-2 & $40.43 \mathrm{abc}$ & $27.24 \mathrm{~d}-\mathrm{g}$ & $45.38 \mathrm{bcd}$ & $39.9 \mathrm{abc}$ & $38.53 \mathrm{ef}$ & 37.19de & $37.54 \mathrm{~b}$ & $27.76 \mathrm{bcd}$ \\
\hline TMO-3 & $42.50 \mathrm{a}$ & $27.82 \mathrm{~d}-\mathrm{g}$ & $47.10 \mathrm{ab}$ & $39.31 \mathrm{a}$ & $43.53 \mathrm{abc}$ & $39.35 \mathrm{cde}$ & $43.63 \mathrm{a}$ & $29.53 a-d$ \\
\hline TMO-T & $39.03 \mathrm{bcd}$ & $24.85 \mathrm{fg}$ & $42.31 \mathrm{c}-\mathrm{f}$ & $33.80 \mathrm{c}$ & $39.40 \mathrm{ef}$ & $41.78 \mathrm{bcd}$ & $36.69 \mathrm{bc}$ & $30.96 \mathrm{ab}$ \\
\hline Ofis-3 & $38.63 \mathrm{~cd}$ & $27.39 \mathrm{~d}-\mathrm{g}$ & $40.71 \mathrm{ef}$ & $39.90 \mathrm{abc}$ & $39.50 \mathrm{ef}$ & $38.58 \mathrm{de}$ & $33.56 \mathrm{efg}$ & $27.83 \mathrm{bcd}$ \\
\hline Ofis-4 & $43.33 \mathrm{a}$ & $27.38 \mathrm{~d}-\mathrm{g}$ & $48.78 \mathrm{ab}$ & $34.80 b c$ & $46.80 \mathrm{a}$ & $37.53 \mathrm{de}$ & $43.31 \mathrm{a}$ & $28.30 \mathrm{a}-\mathrm{d}$ \\
\hline Ofis-8 & $41.73 \mathrm{ab}$ & $37.34 \mathrm{a}$ & $50.83 \mathrm{a}$ & $38.75 \mathrm{a}$ & $40.03 \mathrm{de}$ & $49.96 \mathrm{a}$ & $44.26 \mathrm{a}$ & $31.25 \mathrm{ab}$ \\
\hline Ofis-95 & $37.16 \mathrm{de}$ & $29.54 c-f$ & $47.80 \mathrm{ab}$ & $39.40 \mathrm{a}$ & $38.36 \mathrm{ef}$ & $36.29 \mathrm{de}$ & $35.97 \mathrm{~b}-\mathrm{e}$ & $31.27 \mathrm{ab}$ \\
\hline Ofis-96 & $40.96 \mathrm{abc}$ & $25.84 \mathrm{efg}$ & $47.05 \mathrm{ab}$ & $37.02 \mathrm{abc}$ & $34.26 \mathrm{~g}$ & $40.02 \mathrm{~b}-\mathrm{e}$ & $33.89 \mathrm{~d}-\mathrm{g}$ & $26.2 \mathrm{~d}$ \\
\hline Afyon-95 & $40.66 \mathrm{abc}$ & $36.13 \mathrm{ab}$ & $46.58 \mathrm{abc}$ & $39.76 \mathrm{a}$ & $36.64 f g$ & $44.30 \mathrm{bc}$ & $31.47 \mathrm{~g}$ & $30.53 \mathrm{abc}$ \\
\hline Bolvadin-95 & $40.43 \mathrm{f}$ & $27.96 \mathrm{~d}-\mathrm{g}$ & $38.73 \mathrm{f}$ & $35.06 b c$ & $33.90 \mathrm{~g}$ & $40.67 \mathrm{~b}-\mathrm{e}$ & $36.33 \mathrm{bcd}$ & $31.70 \mathrm{a}$ \\
\hline Tinaztepe & $38.50 \mathrm{~cd}$ & $33.12 \mathrm{abc}$ & $50.16 a$ & $33.73 c$ & $44.56 \mathrm{ab}$ & $38.06 \mathrm{de}$ & $44.61 \mathrm{a}$ & $28.56 a-d$ \\
\hline Anayurt & $35.36 \mathrm{e}$ & $28.76 \mathrm{c}-\mathrm{g}$ & 41.56def & $37.70 \mathrm{ab}$ & $36.26 \mathrm{fg}$ & $41.64 b-e$ & $32.47 \mathrm{fg}$ & $31.36 \mathrm{ab}$ \\
\hline Kemerkaya & $38.60 \mathrm{~cd}$ & $31.42 \mathrm{bcd}$ & $44.94 b-e$ & $33.63 \mathrm{c}$ & $41.40 \mathrm{~b}-\mathrm{e}$ & $38.73 \mathrm{de}$ & $34.29 \mathrm{c}-\mathrm{f}$ & $27.26 \mathrm{~cd}$ \\
\hline Zaferyolu & $42.53 \mathrm{a}$ & 29.59 cde & $47.51 \mathrm{ab}$ & $37.60 \mathrm{ab}$ & $42.90 \mathrm{bcd}$ & $45.25 \mathrm{ab}$ & $43.92 \mathrm{a}$ & $29.26 \mathrm{a}-\mathrm{d}$ \\
\hline Mean $_{\text {Sowing times }}$ & $39.41 \mathrm{~A}$ & $29.25 \mathrm{~B}$ & $45.74 \mathrm{~A}$ & $36.90 \mathrm{~B}$ & 40.37 & 38.78 & $37.87 \mathrm{~A}$ & $29.43 \mathrm{~B}$ \\
\hline Years & \multicolumn{2}{|c|}{$34.3 \mathrm{~B}$} & \multicolumn{2}{|c|}{$41.32 \mathrm{~A}$} & \multicolumn{2}{|c|}{$39.58 \mathrm{~A}$} & \multicolumn{2}{|c|}{$33.65 \mathrm{~B}$} \\
\hline M.square $_{\text {Cultivar }}$ & 32.765 & 43.305 & 36.769 & 14.458 & 570.81 & 42.816 & 67.190 & 8.958 \\
\hline F value Cultivar & $17.81 * *$ & $9.85 * *$ & $8.75 * *$ & $5.95 * *$ & $19.43 * *$ & $7.10 * *$ & $56.37 * *$ & $3.43 *$ \\
\hline C.V $(\%)$ & 3.44 & 7.16 & 4.48 & 4.22 & 3.64 & 6.08 & 2.88 & 5.49 \\
\hline
\end{tabular}

Means in the same columns followed by the same letters are not significantly different as statistically.

*, **: Significant at $\mathrm{P}<0.05$ and $\mathrm{P}<0.01$ probability levels, respectively, ns: non significant 
Table 4. The 1000 seed weight, capsule yield, seed yield, and oil yield of poppy cultivars

\begin{tabular}{|c|c|c|c|c|c|c|c|c|}
\hline \multirow{3}{*}{$\begin{array}{l}\text { Cultivars / } \\
\text { sowing times }\end{array}$} & \multicolumn{4}{|c|}{ Capsule yield $\left(\mathrm{kg} \mathrm{ha}^{-1}\right)$} & \multicolumn{4}{|c|}{ Seed yield (kg ha-1) } \\
\hline & \multicolumn{2}{|c|}{$2012 / 13$} & \multicolumn{2}{|c|}{$2013 / 14$} & \multicolumn{2}{|c|}{$2012 / 13$} & \multicolumn{2}{|c|}{$2013 / 14$} \\
\hline & Autumn & Spring & Autumn & Spring & Autumn & Spring & Autumn & Spring \\
\hline TMO-1 & $801.3 \mathrm{c}$ & $214.0 \mathrm{fg}$ & $918.3 \mathrm{~d}$ & $789.0 \mathrm{ab}$ & $946.7 \mathrm{~b}$ & $302.3 \mathrm{fg}$ & $1084.2 \mathrm{~cd}$ & $910.5 \mathrm{ab}$ \\
\hline TMO-2 & $807.3 \mathrm{c}$ & $204.3 \mathrm{~g}$ & $965.0 \mathrm{~d}$ & $748.2 \mathrm{bc}$ & $923.3 \mathrm{~b}$ & $286.0 \mathrm{~g}$ & $1144.2 \mathrm{c}$ & $890.2 \mathrm{abc}$ \\
\hline TMO-3 & $1039.4 \mathrm{a}$ & $319.3 \mathrm{~g}$ & $1205.1 \mathrm{~b}$ & $744.1 \mathrm{c}$ & $1242.3 \mathrm{a}$ & $438.3 \mathrm{bc}$ & $1393.3 \mathrm{ab}$ & $896.7 \mathrm{abc}$ \\
\hline TMO-T & $707.67 \mathrm{de}$ & $260.6 \mathrm{de}$ & 829.7 e & $673.0 \mathrm{~d}$ & $835.4 \mathrm{bc}$ & 353.7def & $995.8 \mathrm{~cd}$ & 788.5 cde \\
\hline Ofis-3 & $530.0 \mathrm{~g}$ & $266.3 \mathrm{efg}$ & $693.3 \mathrm{f}$ & $497.2 \mathrm{~g}$ & $676.7 \mathrm{de}$ & $346.1 d-g$ & 828.9 e & $630.8 \mathrm{f}$ \\
\hline Ofis-4 & $1034.7 \mathrm{a}$ & $247.3 \mathrm{ef}$ & $1137.3 \mathrm{c}$ & $546.3 \mathrm{e}$ & $1276.3 \mathrm{a}$ & $346.6 \mathrm{~d}-\mathrm{g}$ & $1344.8 \mathrm{~b}$ & $695.7 \mathrm{ef}$ \\
\hline Ofis-8 & $923.2 \mathrm{~b}$ & $455.7 \mathrm{a}$ & $1375.6 \mathrm{a}$ & $830.7 \mathrm{a}$ & $1130.7 \mathrm{a}$ & $573.0 \mathrm{a}$ & $1520.4 \mathrm{a}$ & $962.7 \mathrm{a}$ \\
\hline Ofis-95 & $717.1 \mathrm{de}$ & $285.6 \mathrm{~cd}$ & $945.0 \mathrm{~d}$ & $740.1 \mathrm{c}$ & $825.6 \mathrm{bcd}$ & $421.6 \mathrm{bc}$ & $1099.1 \mathrm{~cd}$ & $817.5 \mathrm{bcd}$ \\
\hline Ofis-96 & $752.0 \mathrm{~d}$ & $311.3 \mathrm{c}$ & $921.3 \mathrm{~d}$ & $501.6 \mathrm{fg}$ & 878.4 bc & $380.6 \mathrm{~cd}$ & $1066.5 \mathrm{~cd}$ & $614.3 \mathrm{f}$ \\
\hline Afyon-95 & $613.3 \mathrm{f}$ & $409.0 \mathrm{~b}$ & $841.7 \mathrm{e}$ & $799.6 \mathrm{a}$ & $740.3 \mathrm{~cd}$ & $533.6 \mathrm{a}$ & $985.3 \mathrm{~d}$ & $920.0 \mathrm{ab}$ \\
\hline Bolvadin-95 & $416.7 \mathrm{~h}$ & $292.6 \mathrm{~cd}$ & $501.0 \mathrm{~g}$ & 540.3 ef & $523.5 \mathrm{e}$ & $375.0 \mathrm{cde}$ & $627.1 \mathrm{f}$ & $650.3 \mathrm{f}$ \\
\hline Tinaztepe & $1045.6 \mathrm{a}$ & $221.0 \mathrm{fg}$ & $1148.3 \mathrm{bc}$ & $736.3 \mathrm{c}$ & $1238.7 \mathrm{a}$ & 312.0efg & $1352.5 b$ & $873.7 \mathrm{abc}$ \\
\hline Anayurt & $449.7 \mathrm{~h}$ & $210.3 \mathrm{fg}$ & $465.3 \mathrm{~g}$ & $552.6 \mathrm{e}$ & $571.8 \mathrm{e}$ & $300.2 \mathrm{fg}$ & $596.7 \mathrm{f}$ & $701.1 \mathrm{def}$ \\
\hline Kemerkaya & $695.3 \mathrm{e}$ & $314.7 \mathrm{c}$ & $793.0 \mathrm{e}$ & 531.0efg & $818.2 \mathrm{bcd}$ & $408.0 \mathrm{bcd}$ & $953.7 \mathrm{de}$ & $629.1 \mathrm{f}$ \\
\hline Zaferyolu & $1043.3 \mathrm{a}$ & $379.6 \mathrm{~b}$ & $1086.0 \mathrm{c}$ & $563.3 \mathrm{e}^{\circ}$ & $1208.3 \mathrm{a}$ & $463.3 \mathrm{~b}$ & $1333.4 \mathrm{~b}$ & $708.8 \mathrm{def}$ \\
\hline Mean $_{\text {Sowing times }}$ & $771.7 \mathrm{~A}$ & $290.1 \mathrm{~B}$ & $921.7 \mathrm{~A}$ & $654.0 \mathrm{~B}$ & $922.3 \mathrm{~A}$ & $389.4 \mathrm{~B}$ & $1088.4 \mathrm{~A}$ & $779.3 \mathrm{~B}$ \\
\hline Years & \multicolumn{2}{|c|}{$531.6 \mathrm{~B}$} & \multicolumn{2}{|c|}{$786.7 \mathrm{~A}$} & \multicolumn{2}{|c|}{$655.8 \mathrm{~B}$} & \multicolumn{2}{|c|}{$933.8 \mathrm{~A}$} \\
\hline M.square $_{\text {Cultivar }}$ & 137427.2 & 17689.9 & 189110.4 & 43434.1 & 184175.6 & 21706.06 & 222489 & 45792.6 \\
\hline F value ${ }_{\text {Cultivar }}$ & $292.95 * *$ & $64.80 * *$ & $229.22 * *$ & $124.05 * *$ & $37.93 * *$ & $27.19 * *$ & $51.19 * *$ & $15.75 * *$ \\
\hline C.V $(\%)$ & 2.80 & 5.69 & 3.11 & 2.87 & 7.55 & 7.25 & 6.05 & 6.91 \\
\hline
\end{tabular}

\begin{tabular}{|c|c|c|c|c|c|c|c|c|}
\hline \multirow{3}{*}{$\begin{array}{l}\text { Cultivars / } \\
\text { sowing times }\end{array}$} & \multicolumn{4}{|c|}{1000 seed weight $(\%)$} & \multicolumn{4}{|c|}{ Oil yield $\left(\mathrm{kg} \mathrm{ha}^{-1}\right)$} \\
\hline & \multicolumn{2}{|c|}{$2012 / 13$} & \multicolumn{2}{|c|}{$2013 / 14$} & \multicolumn{2}{|c|}{$2012 / 13$} & \multicolumn{2}{|c|}{$2013 / 14$} \\
\hline & Autumn & Spring & Autumn & Spring & Autumn & Spring & Autumn & Spring \\
\hline TMO-1 & $0.37 \mathrm{def}$ & $0.32 \mathrm{de}$ & $0.41 \mathrm{abc}$ & $0.28 \mathrm{ab}$ & $369.4 \mathrm{~cd}$ & $120.1 \mathrm{f}$ & $434.3 \mathrm{de}$ & $382.5 \mathrm{ab}$ \\
\hline TMO-2 & $0.34 \mathrm{gh}$ & $0.36 \mathrm{abc}$ & $0.42 \mathrm{ab}$ & $0.24 \mathrm{cde}$ & $384.8 \mathrm{c}$ & $116.5 \mathrm{f}$ & $448.6 \mathrm{dc}$ & $329.2 b-e$ \\
\hline TMO-3 & $0.42 \mathrm{ab}$ & $0.35 \mathrm{a}-\mathrm{d}$ & $0.42 \mathrm{ab}$ & $0.27 b c$ & 484.9ab & $178.8 \mathrm{bcd}$ & $534.1 \mathrm{bc}$ & $370.7 \mathrm{abc}$ \\
\hline TMO-T & $0.38 \mathrm{cde}$ & $0.31 \mathrm{de}$ & $0.39 \mathrm{~cd}$ & $0.28 \mathrm{ab}$ & $336.7 \mathrm{cde}$ & $149.7 \mathrm{def}$ & $411.5 \mathrm{def}$ & $307.6 \mathrm{~d}-\mathrm{g}$ \\
\hline Ofis-3 & $0.38 \mathrm{cde}$ & $0.32 \mathrm{de}$ & $0.38 \mathrm{de}$ & $0.26 \mathrm{bcd}$ & $275.4 \mathrm{efg}$ & $135.1 \mathrm{ef}$ & $345.4 f$ & $227.4 \mathrm{~h}$ \\
\hline Ofis-4 & $0.43 \mathrm{a}$ & $0.38 \mathrm{a}$ & $0.43 \mathrm{a}$ & $0.25 \mathrm{bcd}$ & $535.5 \mathrm{ab}$ & $158.5 \mathrm{cde}$ & $547.2 \mathrm{bc}$ & $299.4 d-g$ \\
\hline Ofis-8 & $0.40 \mathrm{abc}$ & $0.39 \mathrm{a}$ & $0.43 \mathrm{a}$ & $0.31 \mathrm{a}$ & $471.7 \mathrm{~b}$ & $233.5 \mathrm{a}$ & $644.0 \mathrm{a}$ & $356.7 \mathrm{a}-\mathrm{d}$ \\
\hline Ofis-95 & $0.33 \mathrm{~h}$ & $0.30 \mathrm{ef}$ & $0.39 \mathrm{bcd}$ & $0.27 b c$ & $346.7 \mathrm{cde}$ & $187.0 \mathrm{bc}$ & 443.6de & $328.9 \mathrm{~b}-\mathrm{e}$ \\
\hline Ofis-96 & $0.34 \mathrm{fgh}$ & $0.34 \mathrm{bcd}$ & $0.40 \mathrm{bcd}$ & $0.26 \mathrm{bcd}$ & $360.3 \mathrm{~cd}$ & $162.3 \mathrm{cde}$ & $430.2 \mathrm{de}$ & $251.9 \mathrm{gh}$ \\
\hline Afyon-95 & $0.36 \mathrm{efg}$ & $0.37 \mathrm{ab}$ & $0.39 \mathrm{~cd}$ & $0.22 \mathrm{e}$ & 306.0def & $226.1 \mathrm{a}$ & $387.6 \mathrm{ef}$ & $392.5 \mathrm{a}$ \\
\hline Bolvadin-95 & $0.40 \mathrm{bcd}$ & 0.33 cde & $0.39 \mathrm{bcd}$ & $0.24 \mathrm{de}$ & $219.66 \mathrm{~g}$ & $162.7 \mathrm{cde}$ & $259.1 \mathrm{~g}$ & $273.0 \mathrm{e}-\mathrm{h}$ \\
\hline Tinaztepe & $0.39 \mathrm{bcd}$ & $0.26 \mathrm{f}$ & $0.44 \mathrm{a}$ & $0.30 \mathrm{a}$ & $524.7 \mathrm{ab}$ & $142.4 \mathrm{def}$ & $550.2 \mathrm{bc}$ & 367.0abc \\
\hline Anayurt & $0.34 \mathrm{gh}$ & $0.34 b-e$ & $0.35 \mathrm{e}$ & $0.31 \mathrm{a}$ & $250.7 \mathrm{fg}$ & $133.0 \mathrm{ef}$ & $254.8 \mathrm{~g}$ & $306.5 \mathrm{e}-\mathrm{h}$ \\
\hline Kemerkaya & $0.39 \mathrm{bcd}$ & $0.28 \mathrm{f}$ & $0.38 \mathrm{de}$ & $0.24 \mathrm{cde}$ & $346.2 \mathrm{cde}$ & $187.7 \mathrm{bc}$ & 391.3ef & $266.8 \mathrm{fgh}$ \\
\hline Zaferyolu & $0.41 \mathrm{abc}$ & $0.37 \mathrm{ab}$ & $0.42 \mathrm{abc}$ & $0.25 \mathrm{cde}$ & $543.7 \mathrm{a}$ & $213.1 \mathrm{ab}$ & $573.5 \mathrm{ab}$ & $314.5 c-f$ \\
\hline Mean ${ }_{\text {Sowing times }}$ & $0.38 \mathrm{~A}$ & $0.34 \mathrm{~B}$ & $0.40 \mathrm{~A}$ & $0.27 \mathrm{~B}$ & $383.7 \mathrm{~A}$ & $167.1 \mathrm{~B}$ & $446.1 \mathrm{~A}$ & $318.3 \mathrm{~B}$ \\
\hline Years & \multicolumn{2}{|c|}{0.36} & \multicolumn{2}{|c|}{0.34} & \multicolumn{2}{|c|}{$275.4 \mathrm{~B}$} & \multicolumn{2}{|c|}{$382.2 \mathrm{~A}$} \\
\hline M.square Cultivar & 0.0414 & 0.0421 & 0.0018 & 0.0021 & 33134.9 & 4062.406 & 37587.5 & 7378.71 \\
\hline F value Cultivar & $17.82 * *$ & $14.79 * *$ & $8.84 * *$ & $10.21 * *$ & $33.01 * *$ & $15.28 * *$ & $34.71 * *$ & $10.66 * *$ \\
\hline C.V $(\%)$ & 3.38 & 4.98 & 3.57 & 5.42 & 8.25 & 9.75 & 7.37 & 8.26 \\
\hline
\end{tabular}

Means in the same columns followed by the same letters are not significantly different as statistically.

**: significant at $\mathrm{P}<0.01$ probability levels.

\section{Oil content and oil yield}

The mean oil content of the years $(41.9 \%$ and $40.5 \%$, respectively), autumn and spring sowing times $(41.6 \%$ $42.7 \%$ in the first year and $40.0 \%-40.9 \%$ in the second year) was found to be very close to each other (Table 5). In both years (2012-13 and 2013-14), among the cultivars, the highest oil content was determined from the Zaferyolu cultivar (45.0- $46.3 \%$ in the first year and $43.0 \%-44.3 \%$ in the second year) in all the sowing times (Table 4). The highest oil yield was obtained from the Zaferyolu (573.5 $\left.\mathrm{kg} \mathrm{ha}^{-1}\right)$, Ofis- $4\left(535.5 \mathrm{~kg} \mathrm{ha}^{-1}\right)$ Tinaztepe $\left(524.7 \mathrm{~kg} \mathrm{ha}^{-1}\right)$, TMO-3 (484.9 $\left.\mathrm{kg} \mathrm{ha}^{-1}\right)$ and Ofis $8\left(644.0 \mathrm{~kg} \mathrm{ha}^{-1}\right)$ cultivar in the autumn sowing, and Ofis-8 $\left(233.5 \mathrm{~kg} \mathrm{ha}^{-1}\right)$, Afyon95 (392.5 kg ha-1), TMO-1 (382.5 kg ha-1) and Tinaztepe $\left(367.0 \mathrm{~kg} \mathrm{ha}^{-1}\right)$ cultivars in the spring sowing times (Table 4). 


\section{Morphine content}

The mean morphine content in the spring sowing $(0.77 \%)$ was higher than that of the autumn sowing
$(0.70 \%)$. The morphine content of the poppy cultivars ranged from $1.00 \%$ (Ofis- 3 ) to $0.47 \%$ (Kemerkaya) in the autumn sowing and ranged from $0.97 \%$ (TMO-3) to $0.45 \%$ (Kemerkaya) in the spring sowing (Table 5).

Table 5. The oil content and morphine contents of Turkish opium poppy cultivars

\begin{tabular}{|c|c|c|c|c|c|c|c|}
\hline \multirow{3}{*}{$\begin{array}{l}\text { Cultivars / } \\
\text { sowing times }\end{array}$} & \multicolumn{4}{|c|}{ Oil content $(\%)$} & \multicolumn{3}{|c|}{ Morphine content $(\%)+$} \\
\hline & \multicolumn{2}{|c|}{$2012 / 13$} & \multicolumn{2}{|c|}{$2013 / 14$} & \multirow{2}{*}{ Seed colors } & \multicolumn{2}{|c|}{$2012 / 13$} \\
\hline & Autumn & Spring & Autumn & Spring & & Autumn & Spring \\
\hline TMO-1 & $39.0 \mathrm{~d}$ & $39.6 \mathrm{~cd}$ & $40.0 \mathrm{def}$ & $42.2 \mathrm{a}-\mathrm{d}$ & L. brown & $0.67 d-h$ & $0.65 \mathrm{f}$ \\
\hline TMO-2 & $41.7 \mathrm{c}$ & $40.5 \mathrm{bcd}$ & $42.4 \mathrm{abc}$ & $37.1 \mathrm{e}$ & Blue & $0.72 b-f$ & $0.56 \mathrm{~h}$ \\
\hline TMO-3 & $39.2 \mathrm{~d}$ & $40.7 \mathrm{bcd}$ & $38.3 \mathrm{f}$ & $41.3 \mathrm{a}-\mathrm{d}$ & White & $0.76 \mathrm{~b}-\mathrm{e}$ & $0.97 \mathrm{a}$ \\
\hline TMO-T & $40.3 \mathrm{~cd}$ & $42.3 \mathrm{a}-\mathrm{d}$ & $41.3 \mathrm{a}-\mathrm{e}$ & $39.0 \mathrm{de}$ & Blue & $0.85 \mathrm{~b}$ & $0.95 \mathrm{a}$ \\
\hline Ofis-3 & $40.6 \mathrm{~cd}$ & $39.0 \mathrm{~d}$ & $41.6 \mathrm{a}-\mathrm{d}$ & $36.0 \mathrm{e}$ & Blue & $1.00 \mathrm{a}$ & $0.95 \mathrm{a}$ \\
\hline Ofis-4 & $42.5 \mathrm{bc}$ & $45.6 \mathrm{a}$ & $40.6 b-e$ & $43.0 \mathrm{abc}$ & L. brown & $0.56 \mathrm{gh} 1$ & $0.83 \mathrm{c}$ \\
\hline Ofis-8 & $41.6 \mathrm{c}$ & $40.6 \mathrm{bcd}$ & $42.3 \mathrm{abc}$ & $37.0 \mathrm{e}$ & White & $0.84 \mathrm{bc}$ & $0.95 \mathrm{a}$ \\
\hline Ofis-95 & $42.0 \mathrm{bc}$ & $44.3 \mathrm{ab}$ & $40.3 c-f$ & $40.3 \mathrm{~cd}$ & Yellow & $0.56 \mathrm{gh} 1$ & $0.76 \mathrm{e}$ \\
\hline Ofis-96 & $41.0 \mathrm{~cd}$ & $42.6 a-d$ & $40.4 c-f$ & $41.0 \mathrm{bcd}$ & Yellow & $0.69 \mathrm{c}-\mathrm{g}$ & $0.75 \mathrm{e}$ \\
\hline Afyon-95 & $41.3 \mathrm{c}$ & $42.2 \mathrm{a}-\mathrm{d}$ & $39.3 \mathrm{ef}$ & $42.6 a b c$ & Yellow & $0.58 \mathrm{f}-1$ & $0.61 \mathrm{~g}$ \\
\hline Bolvadin-95 & $42.2 \mathrm{bc}$ & $43.4 \mathrm{ab}$ & $41.4 \mathrm{a}-\mathrm{d}$ & $42.4 \mathrm{a}-\mathrm{d}$ & Blue & $0.76 \mathrm{~b}-\mathrm{e}$ & $0.90 \mathrm{~b}$ \\
\hline Tinaztepe & $42.4 b c$ & $45.7 \mathrm{a}$ & $40.7 \mathrm{~b}-\mathrm{e}$ & $42.6 a b c$ & Yellow & $0.78 \mathrm{bcd}$ & $0.79 \mathrm{~d}$ \\
\hline Anayurt & $44.0 \mathrm{ab}$ & $44.3 \mathrm{ab}$ & $42.6 \mathrm{ab}$ & $43.7 \mathrm{ab}$ & Yellow & $0.52 \mathrm{~h} 1$ & $0.66 \mathrm{f}$ \\
\hline Kemerkaya & $42.3 \mathrm{bc}$ & $46.0 \mathrm{a}$ & $41.0 \mathrm{a}-\mathrm{d}$ & $42.5 \mathrm{abc}$ & Yellow & 0.471 & 0.451 \\
\hline Zaferyolu & $45.0 \mathrm{a}$ & $46.3 \mathrm{a}$ & $43.0 \mathrm{a}$ & $44.3 \mathrm{a}$ & White & $0.62 \mathrm{e}-\mathrm{h}$ & $0.88 \mathrm{~b}$ \\
\hline Mean $_{\text {Sowing times }}$ & 41.6 & 42.7 & 40.0 & 40.9 & \multirow{2}{*}{\multicolumn{2}{|c|}{$0.70 \mathrm{~B}$}} & \multirow[t]{2}{*}{$0.77 \mathrm{~A}$} \\
\hline Years & & 1.9 & & & & & \\
\hline M.square Cultivar & 7.565 & 17.174 & 4.926 & 19.546 & & 0.0620 & 0.0789 \\
\hline F value Cultivar & $8.33 * *$ & $6.22 * *$ & $5.43 * *$ & $9.69 * *$ & & $14.44 * *$ & $767.69 * *$ \\
\hline C.V $(\%)$ & 2.28 & 3.87 & 2.32 & 3.47 & & 9.43 & 1.30 \\
\hline
\end{tabular}

Means in the same columns followed by the same letters are not significantly different as statistically.

**: Significant at $\mathrm{P}<0.01$ probability levels. +: The morphine contents were belonging to 2012/13 year

\section{DISCUSSION}

The capsule yield, seed yield, oil yield, plant height, capsule number and capsule width in all poppy cultivars tested in the second year were significantly higher than those of the first year in both the autumn and spring sowings. The great yield differences between years were mainly derived from the higher rainfall in the second year according to the first year. The rainfall in the spring months (March, April, and May) is very important for healthy and strong seedling development (Vanova et al., 2006). The spring season precipitations in the second year in particular were higher than in the first year. The poppy cultivars weren't stressed to high temperatures and water deficit during the tillering periods due to high precipitation in the spring sowing of the second year (Svobodova and Misa, 2004). Therefore, capsule yield and seed yield of the autumn and spring sowing in the second year were close to each other.

The capsule yield, seed yield and yield components in all the poppy cultivars in the autumn sowing were significantly higher than the spring sowing in both years. The mean capsule yield in the autumn sowing was higher by approximately $62.4 \%$ during $2012 / 13$ and $29.0 \%$ during 2013/14, compared to the spring sowing. Similarity, the mean seed yield in the autumn sowing was higher by approximately $57.7 \%$ during 2012/13 and $28.4 \%$ during 2013/14, compared to the spring sowing.
The reason for these differences were significantly higher in the plant characteristics such as plant height, capsule number per plant, capsule width and capsule length in the autumn sowing than in the spring sowing. This increase in the capsule characteristics might be due to longer vegetation periods, more developed roots and the finer development of the plant in the autumn sowing compared to the spring sowing. The first development stage and flowering period in the autumn sowing did not coincide with high temperatures and drought due to the available moisture and nutrient according to the spring sowing. The root system of the poppy is finer and the roots are deeper in a long growing season. In addition, plant growth is better in the long growing period. Yilmaz (1997) reported that capsule yield and seed yield in the autumn sowing was three times higher due to finer development and resistance to diseases compared to the spring sowing. The same authors stated that the fertilization problem and periodically short stress in the spring sowing could be due to dry weather and extreme temperatures. Similarly, Gumuscu (1996) stated that the capsule and seed yield in the autumn sowing was significantly higher according to the spring sowing.

There are large variations in the yields and yield components of the poppy cultivars. In the autumn sowing, while in both capsule yield and seed yield the highest were determined in TMO-3, Ofis-4, Ofis-8, Tinaztepe and 
Zaferyolu cultivars, TMO-1, Ofis-8 and Afyon-95 cultivars in the spring sowing were identified as high yielding cultivars. These differences among the poppy cultivars are due to the genetic potential, the plant characteristics, the root structures and the nutrient uptake capacities of the varieties as reported by previous authors (Gumuscu and Arslan, 1999; Karadavut and Arslan, 2006; Gumuscu et al., 2008; Ipek, 2011). In addition, generally, plant height, capsule number, capsule width, capsule length and 1000 seed weight of the high-yielding cultivars (TMO-3, TMO-T, Ofis-3, Ofis-4, Ofis-8, Afyon-95, Tinaztepe and Zaferyolu) were higher than those of the low-yielding cultivars (Table 3 and 4). The previous researchers reported that the capsule yield and seed yield of the poppy cultivars varied between $61.4-697.2 \mathrm{~kg}$ ha- ${ }^{1}$ and 82.2-767.5 $\mathrm{kg} \mathrm{ha}^{-1}$ (Gumuscu and Arslan, 1999), 450.3-1331.0 and 512.0-1511.0 kg ha-1 (Gumuscu and Arslan, 2008), 2029.7-2781.5 kg ha' and 1088.0-1550.0 $\mathrm{kg} \mathrm{ha}^{-1}$ (Ipek, 2011) $1200.0 \mathrm{~kg} \mathrm{ha}^{-1}$ and $1500.0 \mathrm{~kg} \mathrm{ha}^{-1}$ (Kosar et al., 2012), 92.9-140.5 kg ha-1 and 577.4-1046.4 $\mathrm{kg} \mathrm{ha}^{-1}$ (Boydak and Kavurmac1, 2015). The differences in capsule/seed yield could be the result of climatic and soil conditions, sowing times, agronomic practices and genotypes.

The mean oil content in the years and sowing times (autumn and spring sowing) were found to be very close to each other; however, among the cultivars at the point of the oil content significant differences were observed in both years and sowing times. The differences among the cultivars might be due to the variety of characteristics such as efficiency in the production of the secondary metabolites of the poppy. In the present study, the oil content varied between $36.0 \%$ and $46.3 \%$. Previous studies reported that the oil content of the poppy varied between 40.4-44.7\% (Katar and Yilmaz, 1997), 38.8$53.3 \%$ (Arslan et al., 2000) and 32.4-45.5\% (Ozcan and Atalay, 2006). At the point of the oil yield, the highest oil yield was detected in TMO-1, TMO-3, Ofis-4, Ofis-8, Tinaztepe, Zaferyolu and Afyon-95 cultivars in both years and sowing times (Table 4). At the same time, these cultivars were high-yielding varieties. A significant and positive correlation between the seed yield with oil yield was reported by Sethi et al. (1990).

The main component of the poppy's alkaloids was morphine, and its ratio varied between $0.45 \%$ and $1.00 \%$ depending on the cultivars and sowing times. The morphine content of the industrial poppies was approximately $1 \%$ (Skalicky et al., 2014). In the present research, the morphine contents of TMO-3, Ofis-3, TMOT, Ofis- 8 and Bolvadin- 95 cultivars in the spring sowing were determined as $0.97 \%, 0.95 \%, 0.95 \%, 0.95 \%$, and $0.90 \%$, respectively. At the same time, the morphine contents in the autumn sowing of these cultivars were higher than that of other cultivars. These cultivars could be used for industrial purposes. The mean morphine content in the spring sowed cultivars was higher compared to the autumn sowed ones. The reasons for these differences could be higher synthesize rate of the secondary metabolites due to stress factors such as low moisture and high temperature in the spring sowing. At the same time, among the morphine content of the cultivars significantly differences were found due to the capsule characteristic and genetic structures of the varieties. Yadav et al. (2007) stated that positive correlations were determined between the morphine content with the capsule size, the capsule weight, and the number of capsules per plant. Marculescu and Bobit (2001) and Harvest et al. (2009) confirm that the morphine content correlated with the capsule mass, the growth, and the development of the capsule. Many factors affecting the morphine accumulation during the vegetation periods were noted by Jaszberenyi and Nemeth (2012) and Skalicky et al. (2014). Many researchers determined that the morphine content in the poppy can be affected by the genetic capabilities of the cultivar, the nitrogen nutrition, the water regime, diseases and pests (Marculescu and Bobit, 2001; Harvest et al., 2009; Jaszberenyi and Nemeth, 2012; Skalicky et al., 2014).

\section{CONCLUSION}

The results obtained from the present study indicated that the capsule yield, seed yield, examined yield components, oil content, oil yield and morphine content of the poppy cultivars varied according to years, sowing times and varieties.

As a result, under Isparta's ecological conditions: i) The capsule yield, seed yield and oil yield of the autumn sowing was significantly higher than in the spring sowing.

ii) It could be suggested that TMO-3, Ofis-4, Ofis- 8 , Tinaztepe and Zaferyolu cultivars in the autumn sowing and the TMO-1, Ofis- 8 and Afyon-95 cultivars in the spring sowing because of their higher capsule, seed and oil yields.

iii) The TMO-3, TMO-T, Ofis-3, Ofis- 8 and Bolvadin95 cultivars could be used for industrial purposes due to their higher morphine contents.

\section{ACKNOWLEDGEMENTS}

The author thanks the Afyonkarahisar-Bolvadin Poppy Alkaloid Office for providing the poppy cultivars and contributions to the morphine analysis.

\section{LITERATURE CITED}

Arslan, N., R. Buyukgocmen and A. Gumuscu. 2000. Oil and morphine contents of Turkish poppy populations. J. of Field Crops Research Institute. 9: 56-60 (in Turkish).

Boydak, E. and Z. Kavurmaci. 2015. Adaptation of some of poppy (Papaver somniferum L.) types in the Eastern Gateway Region. Turk J. of Nature and Science. 4: 44-47.

Erdemoglu, N., S. Ozilhan, F. Oztop and B. Sener. 2002. Analaysis with HPLC of alkaloids in poppy capsules sown in Turkey. $14^{\text {th }}$ Herbal Pharmaceutical Raw Materials Conference, 29-31 May 2002, pp: 224-227 (in Turkish).

FAO. 2015. Food and Agriculture Organization of the United Nations-2015.

Gumuscu, A. 1996. A Comparison of yield components between selected varieties and lines in poppy (Papaver somniferum L.) Ankara University Institute of Natural and Applied Sci. Master Thesis, 81p (in Turkish). 
Gumuscu, A. and N. Arslan. 1999. Comparing yield and yield components of some selected poppy (Papaver somniferum L.) lines. Turk J. of Agric. and Forestry. 23: 991-997 (additional number 4) (in Turkish).

Gumuscu, A. and N. Arslan. 2008. Researches on heterosis on yield and yield components of some poppy hybrid (Papaver somniferum L.) lines. Journal of Agric. Science. 14: 365-373 (in Turkish).

Gumuscu, A., N. Arslan and EO. Sarihan. 2008. Evaluation of selected poppy (Papaver somniferum L.) lines by their morphine and other alkaloids contents. European Food Research and Technology. 226: 1213-1220.

Harvest, T., PH. Brown, A. Fist, A. Gracie, D. Gregory and A. Koutoulis. 2009. The latex capacity of opium poppy capsules is fixed early in capsule development and is not a major determinant in morphine yield. Annual Applied Biology. 154: 251-258.

Ipek, G. 2011. The researches on some botonical and agricultural characteristics of selected poppy (Papaver somniferum L.) lines with high morphine. Ankara University Institute of Natural and Applied Sci. Master Thesis, 104p (in Turkish).

Jaszberenyi, C and E. Nemeth. 2012. Connection of frost tolerance and alkaloid accumulation potential in poppy (Papaver somniferum L.). J. Applied Food Quality Botany. 85: 116-119.

Karabuk, B. 2012. The effects of nitrogen fertilization and sowing methods on agricultural and quality of poppy (Papaver somniferum L.) varieties. Ondokuz Mayıs Uni Inst of Nat and Appl Sci PhD Thesis, 120p (in Turkish).

Karadavut, U and N. Arslan. 2006. Some plants characteristics of poppy (Papaver somniferum L.) cultivars and populations with foreign origin. J. of Plants Research. 1: 1-5 (in Turkish)

Katar, D. and G. Yilmaz. 1997. The effects on yield and yield components of poppy of nitrogen application times and doses. Türkey $2^{\text {nd }}$ Field Crops Cong. 22-25 September 1997 (in Turkish).

Koc, H. R. Ulker, A. Gunes, G. Gumuscu, B. Ercan, I. Topal, I. Kara, F. Ozdemir, R. Keles and H. Bayrak. 2012. Determination of some local opium poppy genotypes according to seed and capsule yields. Medicinal and Aromatic Plants Symposium 13-15 September 2012, pp: 255-259 (in Turkish).

Koc, H., A. Gunes, O. Gunduz, R. Ulker, G. Gumuscu and S. Aksoyak. 2014. Evaluation of certain opium poppy genotypes for seed and capsule yields and morphine content under Konya province conditions. Medicinal and Aromatic Plants Symposium 23-25 September 2014, pp: 348-341 (in Turkish).

Kosar, FC., H. Camci A. Kose and O. Bilir. 2012. Geçit Kuşağ Tarımsal Araştırma Enstitüsü Müdürlüğü Tarafından Geliştirilen Yeni Haşhaş Çeşitleri. Medicinal and Aromatic Plants Symposium 13-15 September 2012, pp: 324-328 (in Turkish)

Marculescu, A. and D. Bobit. 2001. Studies on the morphine content of Papaver somniferum L. Roum. Biotec. Letter. 6: 403-409.

Ozcan, MM. and C. Atalay. 2006. Determination of seed and oil properties of some poppy (Papaver somniferum L.) varieties. Grasas Yaceites. 57: 169-174.

Prajapati, S., S. Bajpai, D. Singh, R. Luthra, MM. Gupta and S. Kumar. 2002. Alkaloid profiles of the Indian land races of the opium poppy Papaver somniferum L. Genet. Res. Crop Evoluation. 49: 183-188.

Sethi, KL., RL. Sapra, R. Gupta, KS. Dhindsa and NK. Sangwan. 1990. Performance of poppy cultivars in relation to seed, oil and latex yields under different environments. J. Sci. Food Agriculture. 52: 309-313.

Skalicky, M., V. Hejnak, J. Novak, A. Hejtmankova and I. Stranska. 2014. Evaluation of selected poppy (Papaver somniferum L.) cultivars. Industrial aspect. Turk. J. of Field Crops. 19: 189-196.

Svobodova, I. and P. Misa. 2004. Effect of drought stress on the formation of yield elements in spring barley and the potential of stress expression reduction by foliar application of fertilizers and growth stimulator. Plant Soil Environment. 50: 493-446.

TUIK, 2015. Turkey Statistically Office-2015.

Vanova, M., S. Palik, J. Hajslova and I. Buresova. 2006. Grain quality and yield of spring barley in field trials under variable growing conditions. Plant Soil Environment. 52: 211-219.

Yadav, HK., S. Shukla, A. Rastogi and SP. Singh. 2007. Assessment of diversity in new genetic stock of opium poppy (Papaver somniferum L.). Indian J. Agric. Science. 77: 537-539.

Yilmaz, G. 1997. The investigations on the sowing date of opium poppy (Papaver somniferum L.) in Tokat conditions. Gaziosmanpasa Uni. J. of Agric. Faculty. 14: 105-122 (in Turkish). 